\title{
CAPÍTULO III
}

\section{AVANCES DE LA DIVULGACIÓN DE INFORMACIÓN NO FINANCIERA EN LA INDUSTRIA MANUFACTURERA COLOMBIANA}

\section{Deisy Nohemí Sánchez Villamil}

Contadora Pública y Candidata a Magister en Contabilidad \& Finanzas. Docente investigadora del programa de Contaduría Pública de la Fundación Universitaria Panamericana - Unipanamericana, Sede Bogotá. Líder del Semillero de Investigación SICUP (Semillero de Investigación Contable Unipanamericana). Correo electrónico: dlsanchezv@unipanamericana.edu.co.

https://orcid.org/0000-0002-4057-0687.

\section{Rubén Darío Brand Jaramillo}

Contador Público. Estudiante de Maestría en Administración de Empresas (MBA). Docente investigador del programa de Contaduría Pública de la Fundación Universitaria Panamericana - Unipanamericana, Sede Bogotá. Correo electrónico: rbrand@ unipanamericana.edu.co.

https://orcid.org/0000-0002-0976-6729.

\section{Robinson Daniel Cisnero Ibañez}

Estudiante de sexto semestre del programa de Contaduría Pública de la Fundación Universitaria Panamericana - Unipanamericana, Sede Bogotá. Integrante del semillero SICUP (Semillero de Investigación Contable Unipanamericana). rcisnero@) unipanamericana.edu.co.

https://orcid.org/0000-0003-3702-4658.

\section{Andrea Johanna Enciso Blanco}

Estudiante de sexto semestre del programa de Contaduría Pública de la Fundación Universitaria Panamericana - Unipanamericana, Sede Bogotá. Integrante del semillero SICUP (Semillero de Investigación Contable Unipanamericana). ajenciso@) unipanamericana.edu.co

https://orcid.org/0000-0002-7123-7254.

\section{Resumen}

Caracterizar la elaboración y divulgación de los reportes corporativos en la industria manufacturera colombiana, es el objetivo del presente capítulo, abordando así, los informes de gestión, los informes de sostenibilidad y los reportes integrados que las empresas 
colombianas manufactureras más grandes del país han elaborado. Dicha caracterización se realiza bajo una metodología cualitativa basada en el estudio de caso de veintidós empresas colombianas. Para ello, la técnica de investigación usada es la observación y la revisión periódica de los reportes corporativos anuales publicados por estas empresas; cuyo periodo de observación corresponde a seis años, iniciando en el año 2013 y finalizando en el 2018. En este sentido, este capítulo presenta como resultados los avances de la divulgación de información no financiera de las empresas manufactureras colombianas analizadas, permitiendo concluir que los desafíos para la industria manufacturera en términos de divulgación de información no financiera son retadores puesto que las necesidades de información no financiera son mayores con el pasar de los años.

Palabras Claves: industrias manufactureras, información no financiera, informes de sostenibilidad, reportes corporativos y reportes integrados.

\title{
PROGRESS IN THE DISCLOSURE OF NON-FINANCIAL INFORMATION IN THE COLOMBIAN MANUFACTURING INDUSTRY
}

\begin{abstract}
Characterize the preparation and dissemination of corporate reports in the Colombian manufacturing industry, is the objective of this chapter, thus addressing management reports, sustainability reports and integrated reports that the largest Colombian manufacturing companies in the country have prepared. Said characterization is carried out using a qualitative methodology based on the case study of twenty-two Colombian companies. For this, the research technique used is the observation and periodic review of the annual corporate reports published by these companies; whose observation period corresponds to six years, beginning in 2013 and ending in 2018. In this sense, this chapter presents as results the progress of the disclosure of non-financial information of the Colombian manufacturing companies analyzed, allowing us to conclude that the challenges for the manufacturing industry, in terms of disclosure of non-financial information, they are challenging since the needs for non-financial information are greater over the years.
\end{abstract}

Keywords: corporate reports, integrated report, manufacturing industries, non-financial information and sustainability reports.

\section{Identificación de la investigación}

Este capítulo de libro es resultado del proyecto de investigación titulado: "Los reportes integrados en Colombia: una propuesta metodológica”, el cual fue aprobado en la convocatoria 2018-2019 con el acto administrativo PI382019. Este proyecto de investigación fue liderado por los profesores Deisy Nohemí Sánchez Villamil, Jairo Alonso Rivera Cachope y Rubén Darío Brand Jaramillo y se encuentra financiado por el programa de Contaduría Pública de la Fundación Universitaria Panamericana - Unipanamericana, Sede Bogotá. 


\section{Introducción}

Las necesidades de información no financiera son mayores con el pasar de los años. Las organizaciones se han visto en la tarea de producir información cualitativa que dé cuenta de sus aspectos sociales, económicos y ambientales. Es por ello, que surge la necesidad de evaluar los avances en la producción de esta información, en especial en las empresas manufactureras puesto que estas se han caracterizado por contar con gran cantidad de personal en sus procesos operativos para lograr sus objetivos en el corto, mediano y largo plazo. Es por ello, que el objetivo del presente capítulo es caracterizar la elaboración y divulgación de los reportes corporativos en la industria manufacturera de Colombia, abordando así, los informes de gestión, los informes de sostenibilidad y los reportes integrados que las empresas colombianas más grandes del país durante el periodo 2013 - 2018 han elaborado.

Para ello, este capítulo de libro se estructura en cuatro apartados. El primero realiza una contextualización de la industria manufacturera en Colombia, donde se establecen varias situaciones, a saber: su origen en 1989, la creación de la ANDI, la contribución de la industria al Producto Interno Bruto (PIB) del país, los factores que pueden afectar la productividad del sector en el largo plazo, entre otros.

El segundo apartado presenta el diseño metodológico de esta investigación, donde se establece el enfoque cualitativo del mismo, el objeto de estudio del trabajo, el periodo analizado, la técnica de investigación utilizada, el proceso para recopilar la información y el análisis realizado.

El tercer apartado presenta la caracterización de los reportes corporativos de la industria manufacturera, estableciendo así, las empresas que han elaborado y divulgado informes de gestión, informes de sostenibilidad y reportes integrados en el periodo 2013-2018. De igual forma se analiza la tendencia en la elaboración de estos reportes, comparando su producción en los años analizados. A su vez, se establecen las razones por las cuales algunas de las empresas analizadas no han presentado información no financiera. Finalmente, el cuarto apartado presenta las conclusiones de este trabajo.

\section{Fundamentación teórica}

El sector industrial es el sector secundario de la economía colombiana; allí se abordan las actividades comerciales que se encargan de transformar la materia prima en bienes de consumo. Este sector contempla las actividades relacionadas con la fabricación y producción de textiles, cuero y marroquinería, calzado, cárnicos y pescado, lácteos, bebidas, madera, papel, cartón, caucho, vidrio, hierro, fármacos, joyería, equipos electrónicos, maquinaria, químicos básicos, vehículos automotores, entre algunos otros (Espinel, Aparicio, y Mora, 2018; Grupo de Investigación Cultura Emprendedora, 2008; ANIF, 2016).

El desarrollo del sector industrial en Colombia tuvo su origen a inicios de 1989 con el modelo de sustitución de importaciones, el cual buscaba proteger la producción nacional y estimular el mercado interno en el país. Este modelo de crecimiento impulsó en gran medida la producción nacional de bienes para satisfacer las necesidades del mercado, sin importar cuales 
fueran las consecuencias, entre ellas, los costos elevados, la poca tecnología para tratar las materias primas y la poca maximización en la eficiencia de las operaciones (FAEDIS , 2019).

Así mismo, la solidez de este sector permitió que la actividad industrial se consolidara como gremio económico, dando surgimiento a la Asociación Nacional de Industriales (ANDI), la cual se caracteriza por ser la vocera del gremio industrial ante diversas problemáticas. Actualmente se denomina Asociación Nacional de Empresarios de Colombia y está conformado por empresas que pertenecen a varios sectores económicos, a saber: sector industrial, sector financiero, sector agroindustrial, sector comercial, sector servicios, entre otros. Adicionalmente, esta asociación tiene un servicio transaccional denominado Vicepresidencia de Desarrollo Económico y Competitividad, el cual se encarga de recopilar y generar información económica, empresarial y de competitividad de cara a los cambios económicos que presenta el país.

De esta manera, una de las áreas más grandes del sector industrial es la industria manufacturera, puesto que ha impulsado otros sectores económicos como son la agricultura, los servicios empresariales, los productos mineros y energéticos, los servicios de transporte y las actividades financieras (Acoplásticos, 2014; Uribe, 2014). En este sentido:

Colombia cuenta con una industria manufacturera madura en algunos sectores, relativamente diversificada, de creciente experiencia exportadora, y con una capacidad instalada importante. Es una industria que puede generar empleo de calidad, que fabrica y exporta productos de valor agregado, que propicia impactos transversales en el desarrollo de las regiones del país, y que, dada su naturaleza, genera un efecto dinamizador sobre otras actividades económicas como la agricultura y los servicios. En la última década, la industria manufacturera colombiana se ha transformado. Duplicó su tasa de inversión, triplicó su producto por habitante, y cuadruplicó sus exportaciones. Esta situación ha llevado a que, hoy en día, las empresas industriales de Colombia sean, en términos generales, competitivas frente a sus pares internacionales en eficiencia operativa y calidad de producto (Acoplásticos, 2014, p. 6).

En este sentido, la industria manufacturera se ha caracterizado por ser una de las actividades productivas más representativas para la economía colombiana por dos razones principales. La primera, por su capacidad para generar empleo a gran cantidad de personas, pues como lo afirma la Organización Internacional del Trabajo (OIT), la industria manufacturera comprende un porcentaje significativo en la generación de empleo para América Latina, especialmente para personas con bajos niveles educativos que buscan mejorar sus condiciones de calidad de vida (Ávila, 2019).

El segundo, es por su representación en el Producto Interno Bruto (PIB) del país. Para el año 2018 el PIB de Colombia presentó un gran crecimiento respecto al 2017, ya que registró una tasa de crecimiento del 2,7\%. Este crecimiento es gracias a la diversidad de actividades económicas que se desarrollan en Colombia, pues como lo afirma la Fundación para la Educación Superior y el Desarrollo (FEDESARROLLO): "Los sectores que impulsaron el crecimiento del PIB en 2018 fueron actividades profesionales, científicas y técnicas $(5,0 \%)$, administración pública y defensa $(4,1 \%)$, comercio y transporte $(3,1 \%)$ e industrias manufactureras (2,0\%)" (Fedesarrollo, 2019, p.15). 
Por su parte, la Encuesta Mensual Manufacturera (EMM) del Departamento Administrativo Nacional de Estadística (DANE) establece que las actividades económicas que permitieron el crecimiento de la industria manufacturera para el año 2018 están relacionadas con la refinación del petróleo, la elaboración de bebidas, la fabricación de papel y cartón, y la fabricación de sustancias químicas (FEDESARROLLO, 2019).

Para el primer trimestre del 2019, el DANE presentó el Índice de Producción Industrial (IPI) ${ }^{1}$, el cual tuvo un crecimiento significativo para el país, superando así las estadísticas del año anterior (El Tiempo, 2019; Portafolio, 2019; CVN, 2019; Revista Dinero, 2019). Este crecimiento se debe a cuatro sectores industriales, a saber: la industria manufacturera; la explotación de minas y canteras; el suministro de electricidad y gas; y por último, el tratamiento y distribución del agua (Revista Dinero, 2019).

De esta manera, la ANDI publicó un informe en julio de 2019 acerca de la coyuntura económica del país en donde establecen que Colombia tuvo un gran crecimiento en su Producto Interno Bruto. En la tabla 1, se presentan doce actividades económicas del país, en las cuales once de ellas registraron crecimientos positivos en el primer trimestre del 2019 en relación con el primer trimestre del 2018, siendo el sector de la construcción el único con variaciones negativas.

Al revisar las industrias manufactureras se evidencia que el primer trimestre del año 2019 tuvo un gran crecimiento frente al primer trimestre del 2018, lo cual permite establecer que el sector industrial es un gran impulsador de la economía colombiana puesto que ha generado efectos multiplicadores en otros sectores económicos del país.

Adicionalmente, FEDESARROLLO publica de forma mensual la Encuesta de Opinión del Consumidor (EOC) y la Encuesta de Opinión Empresarial (EOE). Estas encuestas buscan hacer un seguimiento a la percepción de las empresas y a los hogares colombianos acerca de la situación actual y de las expectativas económicas futuras del sector industrial y del sector comercial. Para el sector industrial estas encuestas arrojaron que el Índice de Confianza Industrial (ICI) ha presentado incrementos en el 2018 y que durante el 2019 ha mantenido un registro estable (FEDESARROLLO, 2019).

1 El DANE realiza cálculos mensuales para ver la evolución del sector industrial en relación con la producción nacional del país, esto se conoce como el Índice de Producción Industrial (IPI). Este índice comprende los sectores minero energético, manufacturero, suministro de electricidad y gas, y captación, tratamiento y distribución de agua (DANE, 2019). 
Tabla 1. Comparativo del PIB para el primer trimestre de 2018 - 2019.

\begin{tabular}{|c|c|c|c|c|c|c|c|}
\hline \multirow{2}{*}{$\begin{array}{c}\text { Colombia: Crecimiento } \\
\text { Económico }\end{array}$} & \multirow{2}{*}{$\begin{array}{c}\text { Part. } \\
\%\end{array}$} & \multicolumn{5}{|c|}{2018} & \multirow{2}{*}{$\begin{array}{c}2019 \\
\text { Trim I }\end{array}$} \\
\hline & & Trim I & Trim II & Trim III & Trim IV & Año & \\
\hline $\begin{array}{c}\text { Agricultura, ganadería, caza, } \\
\text { silvicultura y pesca }\end{array}$ & 6,2 & 1,7 & 5,2 & 0,8 & 1,1 & 2,1 & 1,4 \\
\hline $\begin{array}{l}\text { Explotación de minas y } \\
\text { canteras }\end{array}$ & 5,1 & $-3,5$ & $-0,6$ & 3,2 & 0,0 & $-0,2$ & 5,3 \\
\hline Industrias manufactureras & 12,1 & $-2,1$ & 4,1 & 2,3 & 2,5 & 1,8 & 2,9 \\
\hline $\begin{array}{l}\text { Electricidad, gas, agua, } \\
\text { saneamiento ambiental }\end{array}$ & 3,0 & 2,3 & 2,8 & 3,2 & 2,6 & 2,7 & 3,1 \\
\hline Construcción & 7,0 & $-0,9$ & $-4,9$ & 3,6 & 4,5 & 0,8 & $-5,6$ \\
\hline $\begin{array}{l}\text { Comercio, reparaciones, } \\
\text { transporte, almacenamiento, } \\
\text { alojamiento, servicios de } \\
\text { comida }\end{array}$ & 17,1 & 3,9 & 3,8 & 2,5 & 3,2 & 3,3 & 4,0 \\
\hline Información y comunicaciones & 2,9 & $-0,2$ & 3,1 & 5,1 & 4,0 & 3,0 & 3,9 \\
\hline $\begin{array}{l}\text { Actividades financieras y de } \\
\text { seguros }\end{array}$ & 4,6 & 4,9 & 3,4 & 2,0 & 2,8 & 3,3 & 5,5 \\
\hline Actividades inmobiliarias & 8,9 & 2,2 & 2,0 & 2,0 & 1,8 & 2,0 & 3,0 \\
\hline $\begin{array}{l}\text { Actividades profesionales } \\
\text { científicas y técnicas, servicios } \\
\text { administrativos y de apoyo }\end{array}$ & 7,0 & 7,3 & 6,2 & 4,0 & 3,0 & 5,0 & 3,0 \\
\hline $\begin{array}{c}\text { Administración pública y } \\
\text { defensa; seguridad social, } \\
\text { educación, salud humana y de } \\
\text { servicios sociales }\end{array}$ & 14,7 & 4,5 & 4,7 & 4,0 & 3,7 & 4,2 & 3,3 \\
\hline $\begin{array}{c}\text { Actividades artísticas, de } \\
\text { entretenimiento recreación, } \\
\text { hogares }\end{array}$ & 2,4 & 1,2 & 2,0 & 1,1 & 2,6 & 1,7 & 2,1 \\
\hline Valor agregado bruto & 90,9 & 2,0 & 2,9 & 2,6 & 2,7 & 2,6 & 2,8 \\
\hline $\begin{array}{l}\text { Impuestos menos subvenciones } \\
\text { sobre los productos }\end{array}$ & 9,1 & 1,8 & 2,7 & 2,3 & 2,4 & 2,3 & 2,6 \\
\hline Producto interno bruto & 100,0 & 2 & 2,9 & 2,6 & 2,7 & 2,6 & 2,8 \\
\hline
\end{tabular}

Fuente: Vicepresidencia de Desarrollo Económico y Competitividad, 2019.

De igual forma, FEDESARROLLO ha realizado proyecciones del crecimiento económico del país hasta el 2023, donde se espera que la industria manufacturera presente un crecimiento significativo debido a un mejor desempeño en la producción de petróleo y en la industria textil. Sin embargo, para lograr estos crecimientos se hace necesario optimizar los procesos en términos de innovación y tecnología, pues como lo afirma FEDESARROLLO, "será esencial implementar políticas que contribuyan a mejorar la productividad y a impulsar la innovación en las diferentes actividades manufactureras" ((FEDESARROLLO, 2019, p. 28). 
En este sentido, algunos académicos afirman que la innovación es un factor de primer orden en el desarrollo social y económico de los países, estableciendo que el papel de la innovación y el desarrollo tecnológico son variables que están relacionadas con la productividad y la competitividad. De este modo, para Arraut (2010), la innovación en la economía "es la clave para la alta productividad y la gran prosperidad de todos. Es importante en todos los sectores de la economía y en las empresas tanto manufactureras como de servicio" (p. 27). Por ello, si las organizaciones quieren ampliar su mercado deben implementar modelos de gestión transformadores que les permitan crear procesos y técnicas retadoras, con los cuales desarrollarán mejores estrategias organizacionales.

Sin embargo, estudios revelan que gracias a los acuerdos comerciales que ha realizado el gobierno colombiano y a la posición estratégica del país, Colombia se ha convertido en un punto central para la producción y exportación de bienes. De igual forma, factores como los costos competitivos, la mano de obra calificada, los procesos de producción de alta calidad y la innovación en los productos hacen de Colombia un país destacado internacionalmente en la industria manufacturera (Procolombia, 2019).

A pesar de ello, la industria manufacturera en Colombia depende de algunos factores que pueden afectar su actividad productiva, como lo son: la alta volatilidad de la tasa de cambio, la competencia del mercado internacional, la calidad de las materias primas, los altos costos de la energía, las inflexibilidades del mercado laboral, los elevados costos de transporte y logística, las altas tasas del sistema tributario, el contrabando y la informalidad, entre algunos otros (FAEDIS , 2019; Acoplásticos, 2014). Es por ello, que Acoplásticos² en el año 2014, desarrolló un diagnóstico acerca de las posibles soluciones que permitirán que la industria manufacturera logre superar los obstáculos frente a los factores mencionados anteriormente ${ }^{3}$.

En este sentido, se puede establecer que la industria manufacturera colombiana ha presentado grandes aportes para la economía del país, contribuyendo así al incremento del PIB en Colombia. Sin embargo, al igual que otros sectores económicos, la industria manufacturera se encuentra vulnerable ante factores externos que pueden afectar su productividad. Esto demuestra los grandes retos del país, especialmente de las empresas y del gobierno colombiano para afrontarlas y superarlas, permitiendo así, el mejoramiento productivo en todos los sectores económicos colombianos.

\section{Metodología}

La metodología que se desarrolla en los próximos capítulos es de carácter cualitativo (Delgado y Gutiérrez, 1997), pues lo que se busca es caracterizar la elaboración de los

2 Acoplásticos es el gremio de empresarios de la industria plástica del país. Fue fundada en 1961 y se encuentra conformada por la industria del plástico, caucho, pinturas, tintas, fibras, petroquímica y sus relacionados (Acoplásticos, 2019).

3 Las soluciones que propone Acoplásticos frente a estos obstáculos son: i) disminuir los costos de energía; ii) reducir los sobrecostos de la regulación laboral (parafiscales, liquidaciones, indemnizaciones, incapacidades, entre otros); iii) disminuir tiempos y costos de transporte y logística; iv) disminuir las tasas del sistema tributario; v) fomentar la ciencia, tecnología e innovación; y vi) buscar oportunidad de mercado para la industria colombiana. Frente a estos obstáculos, Acoplásticos desarrolló cincuenta recomendaciones de carácter político (2014). 
reportes corporativos (informes de gestión, informes de sostenibilidad y reportes integrados) en la industria manufacturera en Colombia.

Para ello, la técnica de investigación usada es la observación y la revisión periódica de los reportes corporativos anuales publicados por las empresas analizadas pertenecientes a este sector.

El periodo de observación de estas publicaciones corresponde a seis años, iniciando en el año 2013, fecha en la cual se publica el Marco Internacional de Reporte Integrado hasta el año 2018.

Las empresas que son el objeto de estudio de esta investigación fueron seleccionadas gracias al informe titulado: "Comportamiento de las 1000 empresas más grandes del sector real, por ingresos operacionales" que publicó la Superintendencia de Sociedades en el mes de mayo del presente año (2019) en su página web ${ }^{4}$. Este estudio es un análisis de las 1.000 empresas más grandes de Colombia según sus ingresos operacionales a 31 de diciembre de 2018.

En este sentido, la Superintendencia de Sociedades publicó esta información en un archivo en formato xml, donde se establece información más específica de las empresas, entre ellas, el número de identificación tributario (NIT), la entidad supervisora, el departamento de domicilio, el macro sector al cual pertenecen, el código CIIU y el grupo NIIF al cual estas empresas pertenecen. Adicionalmente, en este archivo se encuentra la información contable de estas empresas a 31 de diciembre de 2018 distribuida en cinco columnas (ingresos operacionales, resultado del periodo, total de activos, total pasivos y total patrimonio). De igual forma, esta base de datos establece la misma información contable a 31 de diciembre de 2017, permitiendo así contrastar y comparar las cifras reportadas por estas empresas para analizar su posicionamiento en el mercado.

Los criterios usados para depurar la información del archivo mencionado y establecer las empresas que fueron objeto de estudio de esta investigación estuvieron enfocados a seleccionar las cien (100) empresas que encabezan este informe, las cuales son las empresas más grandes de Colombia a 31 de diciembre de 2018.

Una vez fueron seleccionadas las empresas, se realizó la clasificación de estas empresas según los sectores económicos a los cuales pertenecen, para esto el criterio de selección fue la columna titulada "Macrosector" de la base de datos de la Superintendencia de Sociedades. Allí se seleccionó el macrosector correspondiente a las empresas manufactureras; las empresas resultantes en este proceso fueron veintidós.

Una vez fueron identificadas estas veintidós empresas, se realizó la búsqueda de los reportes corporativos que estás han elaborado en el periodo de observación 2013 2018. Como se mencionó anteriormente, este periodo fue determinado con base en la publicación del Marco Internacional del Reporte Integrado, pues es en el año 2013 cuando se establecieron los parámetros para su elaboración.

La búsqueda de los reportes corporativos anuales de estas empresas se realiza de dos formas: la primera, en la página web de cada una de estas; la segunda, en la base de datos de divulgación del $\mathrm{GRI}^{5}$, la cual proporciona acceso gratuito a los informes de sostenibilidad

4 Para más información revisar la página web https://www.supersociedades.gov.co/SitePages/ Inicio.aspx

5 Para más información, ver https://database.globalreporting.org/ 
publicados por las empresas. La información recogida en este proceso es clasificada en cuatro categorías, a saber: NP (Reporte No Presentado), IG (Informe de Gestión), RI (Reporte Integrado) e IS (Informe de Sostenibilidad).

Una vez es captada la información de la elaboración y divulgación de los reportes corporativos en las empresas más grandes de Colombia en el 2018 que pertenecen a la industria manufacturera, se realizan los análisis correspondientes para establecer las tendencias de este sector en la elaboración y divulgación de información no financiera.

\section{Resultados}

En la tabla 2, se realiza una presentación de los aspectos generales de las veintidós empresas analizadas pertenecientes a la industria manufacturera; la primera columna hace referencia a la posición de estas en el listado de las mil empresas más grandes del país según el estudio realizado por la Superintendencia de Sociedades (2019). Así mismo, se puede identificar que las ciudades de domicilio donde estas empresas están ubicadas contemplan las regiones más grandes del país.

Tabla 2. Descripción de las empresas pertenecientes a la industria manufacturera.

\begin{tabular}{|c|c|c|c|c|}
\hline No. & Razón Social & $\begin{array}{l}\text { Ciudad } \\
\text { Domicilio }\end{array}$ & Código CIIU - Actividad económica & $\begin{array}{c}\text { Año de } \\
\text { Constitución }\end{array}$ \\
\hline 3 & $\begin{array}{c}\text { Refinería de } \\
\text { Cartagena S.A.S. }\end{array}$ & $\begin{array}{l}\text { Cartagena- } \\
\text { Bolívar }\end{array}$ & $\begin{array}{c}\text { C1921 - Fabricación de productos de la } \\
\text { refinación del petróleo }\end{array}$ & 1957 \\
\hline 12 & Bavaria S.A. & $\begin{array}{l}\text { Bogotá- } \\
\text { Bogotá D.C. }\end{array}$ & $\begin{array}{c}\text { C1103 - Producción de malta, } \\
\text { elaboración de cervezas y otras bebidas } \\
\text { malteadas }\end{array}$ & 1879 \\
\hline 37 & $\begin{array}{c}\text { Sociedad de } \\
\text { Fabricación de } \\
\text { Automotores SAS }\end{array}$ & $\begin{array}{l}\text { Envigado- } \\
\text { Antioquia }\end{array}$ & $\begin{array}{l}\text { C2910 - Fabricación de vehículos } \\
\text { automotores y sus motores }\end{array}$ & 1970 \\
\hline 40 & $\begin{array}{l}\text { General Motors } \\
\text { Colmotores S.A } \\
\end{array}$ & $\begin{array}{c}\text { Bogotá- } \\
\text { Bogotá D.C. }\end{array}$ & $\begin{array}{c}\text { C2910 - Fabricación de vehículos } \\
\text { automotores y sus motores }\end{array}$ & 1956 \\
\hline 46 & $\begin{array}{l}\text { Polipropileno del } \\
\text { Caribe S.A. }\end{array}$ & $\begin{array}{l}\text { Cartagena- } \\
\text { Bolívar }\end{array}$ & $\begin{array}{l}\text { C2013 - Fabricación de plásticos en } \\
\text { formas primarias }\end{array}$ & 1989 \\
\hline 53 & $\begin{array}{c}\text { Cementos Argos } \\
\text { S.A }\end{array}$ & $\begin{array}{l}\text { Barranquilla- } \\
\text { Atlántico }\end{array}$ & $\begin{array}{c}\text { C2394 - Fabricación de cemento, cal } \\
\text { y yeso }\end{array}$ & 1934 \\
\hline 54 & Quala S.A & $\begin{array}{c}\text { Bogotá- } \\
\text { Bogotá D.C. }\end{array}$ & $\begin{array}{c}\text { C1089 - Elaboración de otros productos } \\
\text { alimenticios n.c.p. }\end{array}$ & 1980 \\
\hline 58 & $\begin{array}{c}\text { Alpina Productos } \\
\text { Alimenticios S A }\end{array}$ & $\begin{array}{c}\text { Sopo- } \\
\text { Cundinamarca } \\
\end{array}$ & $\begin{array}{c}\text { C1040 - Elaboración de productos } \\
\text { lácteos }\end{array}$ & 1945 \\
\hline 59 & Postobón SA & $\begin{array}{l}\text { Medellín- } \\
\text { Antioquia }\end{array}$ & $\begin{array}{l}\text { C1104 - Elaboración de bebidas no } \\
\text { alcohólicas, producción de aguas } \\
\text { minerales y de otras aguas embotelladas }\end{array}$ & 1904 \\
\hline
\end{tabular}

Fuente: adaptado de Superintendencia de Sociedades (2019). 
Tabla 2. Descripción de las empresas pertenecientes a la industria manufacturera. (Continuación).

\begin{tabular}{|c|c|c|c|c|}
\hline No. & Razón Social & $\begin{array}{c}\text { Ciudad } \\
\text { Domicilio }\end{array}$ & Código CIIU - Actividad económica & $\begin{array}{c}\text { Año de } \\
\text { Constitución }\end{array}$ \\
\hline 62 & Contegral S.A.S & $\begin{array}{l}\text { Envigado- } \\
\text { Antioquia }\end{array}$ & $\begin{array}{l}\text { C1090 - Elaboración de alimentos } \\
\text { preparados para animales }\end{array}$ & 1951 \\
\hline 63 & $\begin{array}{c}\text { Alimentos } \\
\text { Cárnicos S.A.S. }\end{array}$ & Yumbo-Valle & $\begin{array}{l}\text { C1011 - Procesamiento y conservación } \\
\text { de carne y productos cárnicos }\end{array}$ & 1935 \\
\hline 69 & $\begin{array}{l}\text { Cemex Colombia } \\
\text { S.A. }\end{array}$ & $\begin{array}{l}\text { Bogotá- } \\
\text { Bogotá D.C. }\end{array}$ & $\begin{array}{c}\text { C2394 - Fabricación de cemento, cal } \\
\text { y yeso }\end{array}$ & 1906 \\
\hline 74 & $\begin{array}{c}\text { Industria } \\
\text { Nacional de } \\
\text { Gaseosas S.A. }\end{array}$ & $\begin{array}{l}\text { Bogotá- } \\
\text { Bogotá D.C. }\end{array}$ & $\begin{array}{l}\text { C1104 - Elaboración de bebidas no } \\
\text { alcohólicas, producción de aguas } \\
\text { minerales y de otras aguas embotelladas }\end{array}$ & 1927 \\
\hline 77 & Colombina S A & $\begin{array}{c}\text { Zarzal-Valle } \\
\text { del Cauca }\end{array}$ & $\begin{array}{l}\text { C1082 - Elaboración de cacao, } \\
\text { chocolate y productos de confitería }\end{array}$ & 1918 \\
\hline 78 & $\begin{array}{c}\text { Nestlé de } \\
\text { Colombia S.A }\end{array}$ & $\begin{array}{c}\text { Bogotá- } \\
\text { Bogotá D.C. }\end{array}$ & $\begin{array}{l}\text { C1089 - Elaboración de otros productos } \\
\text { alimenticios n.c.p. }\end{array}$ & 1944 \\
\hline 84 & Grupo Argos S.A. & $\begin{array}{l}\text { Medellín- } \\
\text { Antioquia }\end{array}$ & $\begin{array}{c}\text { K6613 - Otras actividades relacionadas } \\
\text { con el mercado de valores }\end{array}$ & 1934 \\
\hline 86 & Solla S. A. & $\begin{array}{l}\text { Medellín- } \\
\text { Antioquia }\end{array}$ & $\begin{array}{c}\text { C1090 - Elaboración de alimentos } \\
\text { preparados para animales }\end{array}$ & 1948 \\
\hline 91 & Italcol S.A. & $\begin{array}{c}\text { Bogotá- } \\
\text { Bogotá D.C. }\end{array}$ & $\begin{array}{l}\text { C1090 - Elaboración de alimentos } \\
\text { preparados para animales }\end{array}$ & 1970 \\
\hline 92 & $\begin{array}{l}\text { Mexichem } \\
\text { Resinas Colombia } \\
\text { S.A.S. } \\
\end{array}$ & $\begin{array}{l}\text { Cartagena- } \\
\text { Bolívar }\end{array}$ & $\begin{array}{l}\text { C2013 - Fabricación de plásticos en } \\
\text { formas primarias }\end{array}$ & 1965 \\
\hline 96 & $\begin{array}{l}\text { Productos Familia } \\
\text { S A }\end{array}$ & $\begin{array}{l}\text { Medellín- } \\
\text { Antioquia }\end{array}$ & $\begin{array}{l}\text { C1709 - Fabricación de otros artículos } \\
\text { de papel y cartón }\end{array}$ & 1958 \\
\hline 97 & $\begin{array}{l}\text { Colgate Palmolive } \\
\text { Compañía }\end{array}$ & Cali-Valle & $\begin{array}{c}\text { C2023 - Fabricación de jabones y } \\
\text { detergentes, preparados para limpiar y } \\
\text { pulir; perfumes y preparados de tocador }\end{array}$ & - \\
\hline 98 & $\begin{array}{l}\text { Alimentos Finca } \\
\text { S.A.S. }\end{array}$ & $\begin{array}{l}\text { Envigado- } \\
\text { Antioquia }\end{array}$ & $\begin{array}{l}\text { C1090 - Elaboración de alimentos } \\
\text { preparados para animales }\end{array}$ & - \\
\hline
\end{tabular}

Fuente: adaptado de Superintendencia de Sociedades (2019).

Así mismo, se identifica que las actividades económicas que desarrollan estas empresas son diversas lo que permite confirmar la amplitud de la industria manufacturera, abordando así, la fabricación de vehículos, alimentos, bebidas, plásticos, productos derivados del petróleo, entre otros. Adicionalmente, se evidencia el año de constitución de estas empresas, donde se establece que la mitad de estas fueron constituidas durante la primera mitad del siglo XX, lo cual permite determinar que estas empresas tienen una amplia trayectoria en el mercado, puesto que la empresa analizada con menor trayectoria fue creada en el año 1989, teniendo así, treinta años de experiencia en el mundo organizacional. 
Una vez se identificaron estas empresas se realizó la búsqueda de los reportes corporativos que éstas han elaborado en el periodo de observación 2013 - 2018. En este punto, es importante establecer nuevamente que el periodo seleccionado se debe principalmente por la publicación del Marco Internacional de Reporte Integrado en el 2013.

A partir de allí, se realizó la búsqueda de los reportes corporativos anuales de estas empresas de dos formas: la primera, en la página web de cada una de estas; la segunda, en la base de datos de divulgación del GRI. Los resultados de este proceso de búsqueda son clasificados y presentados en la tabla 3, bajo cuatro categorías, a saber: NP (Reporte No Presentado), IG (Informe de Gestión), RI (Reporte Integrado) e IS (Informe de Sostenibilidad). En este sentido, la tabla permite evidenciar que la elaboración de reportes corporativos de las empresas colombianas más grandes del país pertenecientes a la industria manufacturera ha sido diversa. Por ello, fue necesario cuantificar las cuatro categorías de reportes corporativos y sus resultados. Ver tablas 3 y 4 respectivamente.

Tabla 3. Elaboración de Reportes Corporativos en las empresas colombianas de la Industria Manufacturera.

\begin{tabular}{cccccccc}
\hline No. & Razón social & $\mathbf{2 0 1 3}$ & $\mathbf{2 0 1 4}$ & $\mathbf{2 0 1 5}$ & $\mathbf{2 0 1 6}$ & $\mathbf{2 0 1 7}$ & $\mathbf{2 0 1 8}$ \\
\hline 1 & Refinería de Cartagena S.A.S. & IG & IG & RI & IS & RI & IS \\
\hline 2 & Bavaria S.A. & IS & IS & IS & IS & NP & IS \\
\hline 3 & Sociedad de Fabricación de Automotores SAS & IS & IS & IS & IS & IS & IS \\
\hline 4 & General Motors Colmotores S.A & NP & NP & NP & NP & NP & NP \\
\hline 5 & Polipropileno del Caribe S.A. & NP & IS & IS & IS & IS & IS \\
\hline 6 & Cementos Argos S.A & RI & RI & RI & RI & RI & RI \\
\hline 7 & Quala S.A & NP & NP & NP & NP & NP & NP \\
\hline 8 & Alpina Productos Alimenticios S A & IS & IS & IS & IS & IS & IS \\
\hline 9 & Postobón SA & IG & IS & IS & IS & IS & IS \\
\hline 10 & Contegral S.A.S & NP & NP & NP & NP & NP & NP \\
\hline 11 & Alimentos Cárnicos S.A.S. & NP & NP & NP & NP & NP & NP \\
\hline 12 & Cemex Colombia S.A. & IS & IS & IS & IS & RI & RI \\
\hline 13 & Industria Nacional de Gaseosas S.A. & NP & NP & NP & NP & NP & NP \\
\hline 14 & Colombina S A & IS & IS & IS & IS & IS & IS \\
\hline 15 & Nestlé de Colombia S.A & NP & NP & NP & NP & NP & NP \\
\hline 16 & Grupo Argos S.A. & RI & RI & RI & RI & RI & RI \\
\hline 17 & Solla S. A. & NP & NP & NP & NP & NP & NP \\
\hline 18 & Italcol S.A. & NP & NP & NP & NP & NP & NP \\
\hline 19 & Mexichem Resinas Colombia S.A.S. & NP & NP & NP & NP & NP & NP \\
\hline 20 & Productos Familia S A & IG & IS & IS & IS & IS & RI \\
\hline 21 & Colgate Palmolive Compañía & IS & IS & IS & IS & IS & IS \\
\hline 22 & Alimentos Finca S.A.S. & NP & NP & NP & NP & NP & NP \\
\hline
\end{tabular}

Fuente: elaboración propia (2019). 
Tabla 4. Caracterización de los Reportes Corporativos en las empresas colombianas de la Industria Manufacturera.

\begin{tabular}{|c|c|c|c|c|c|}
\hline No. & Razón social & IG & IS & RI & NP \\
\hline 1 & Refinería de Cartagena S.A.S. & 2 & 2 & 2 & \\
\hline 2 & Bavaria S.A. & & 5 & & 1 \\
\hline 3 & Sociedad de Fabricación de Automotores SAS & & 6 & & \\
\hline 4 & General Motors Colmotores S.A & & & & 6 \\
\hline 5 & Polipropileno del Caribe S.A. & & 5 & & 1 \\
\hline 6 & Cementos Argos S.A & & & 6 & \\
\hline 7 & Quala S.A & & & & 6 \\
\hline 8 & Alpina Productos Alimenticios S A & & 6 & & \\
\hline 9 & Postobón SA & 1 & 5 & & \\
\hline 10 & Contegral S.A.S & & & & 6 \\
\hline 11 & Alimentos Cárnicos S.A.S. & & & & 6 \\
\hline 12 & Cemex Colombia S.A. & & 4 & 2 & \\
\hline 13 & Industria Nacional de Gaseosas S.A. & & & & 6 \\
\hline 14 & Colombina S A & & 6 & & \\
\hline 15 & Nestlé de Colombia S.A & & & & 6 \\
\hline 16 & Grupo Argos S.A. & & & 6 & \\
\hline 17 & Solla S. A. & & & & 6 \\
\hline 18 & Italcol S.A. & & & & 6 \\
\hline 19 & Mexichem Resinas Colombia S.A.S. & & & & 6 \\
\hline 20 & Productos Familia S A & 1 & 4 & 1 & \\
\hline 21 & Colgate Palmolive Compañía & & 6 & & \\
\hline 22 & Alimentos Finca S.A.S. & & & & 6 \\
\hline & Total & 4 & 49 & 17 & 62 \\
\hline
\end{tabular}

Fuente: elaboración propia (2019).

En este sentido, al revisar los reportes corporativos de las veintidós (22) empresas que fueron seleccionadas, se esperaba encontrar ciento treinta y dos (132) reportes corporativos en los seis años determinados. Sin embargo, en la tabla 4, se evidencia que los resultados fueron inferiores, dado que la categoría que prevalece es la de "Reporte no presentado".

Por ello, se analizó la presentación de reportes corporativos en cada una de las empresas objeto de estudio, de tal forma que se pudiera identificar cuáles de ellas han dejado de presentar reportes corporativos en al menos un año y cuales nunca lo han hecho.

Allí se encontró que dos empresas (Bavaria S.A. y Polipropileno del Caribe S.A.) dejaron de presentar reportes corporativos en al menos un año; y que, por el contrario, fueron diez las empresas (General Motors Colmotores S.A, Quala S.A, Contegral S.A.S., Alimentos Cárnicos S.A.S., Industria Nacional de Gaseosas S.A., Nestlé de Colombia S.A., Solla S. A., Italcol S.A., Mexichem Resinas Colombia S.A.S. y Alimentos Finca 
S.A.S.) que no presentaron reportes corporativos en ningún año del periodo analizado. Al revisar estas diez empresas se encontraron las razones por las cuales estas no han elaborado reportes corporativos.

La empresa General Motors Colmotores S.A es una entidad colombiana que se encuentra controlada por la empresa estadounidense General Motors Company desde 1979. Al revisar los reportes corporativos de esta última organización se encontró que esta elabora informes de sostenibilidad de manera internacional; para el caso de Colombia la empresa General Motors Colmotores S.A., no elabora ningún reporte corporativo. En este sentido, dado que el presente documento pretende caracterizar la elaboración y divulgación de los reportes corporativos de la industria manufacturera en Colombia los informes elaborados por la empresa estadounidense no son tomados en cuenta.

Quala S.A., es una multinacional colombiana con cobertura en países como Ecuador, México, República Dominicana, Perú y algunos otros países de Centroamérica. Sin embargo, en su página web no se encontraron reportes corporativos. Adicionalmente, al buscar en la base de datos de divulgación del GRI esta empresa no presentó resultados.

Contegral S.A.S., es una empresa de alimentos que está controlada por el Grupo Empresarial Bios, el cual elabora reportes corporativos acerca de su gestión. Sin embargo, Contegral S.A.S. como empresa individual no realiza esta actividad.

Alimentos Cárnicos S.A.S., es una empresa de productos cárnicos que está controlada por el Grupo Empresarial Nutresa, el cual elabora reportes integrados. Sin embargo, al igual que Contegral S.A.S., la empresa Alimentos Cárnicos S.A.S. no elabora reportes corporativos.

Industria Nacional de Gaseosas S.A., se caracteriza por ser una subsidiaria de la empresa multinacional mexicana Coca-Cola FEMSA desde el año 2003 (EMIS Professional, 2019). Por ello, la empresa Industria Nacional de Gaseosas S.A. como entidad sucursal no presenta reportes corporativos.

Nestlé de Colombia S.A. está controlada por la empresa multinacional suiza Nestlé S.A. Por ello, Nestlé de Colombia S.A. no elabora reportes corporativos. Sin embargo, al revisar la base de datos de divulgación del GRI se encontró que Nestlé Berhad (Malasia), Nestlé Bulgaria, Nestlé Grecia, Nestlé Indonesia, Nestlé Filipinas, Nestlé Rusia y Nestlé Estados Unidos, sí elaboran informes de sostenibilidad, algunas de ellas desde el 2007. Esto evidencia el poco avance de Nestlé Colombia S.A. frente a la elaboración y divulgación de información no financiera.

Solla S. A., Italcol S.A. y Alimentos Finca S.A.S. no presentan reportes corporativos en su página web, como tampoco existen resultados de estas en la base de datos de divulgación del GRI. Este hecho permite crear cuestionamientos acerca de las razones por las cuales estas no se han preocupado por elaborar reportes corporativos de su gestión de cara a la sostenibilidad empresarial, resaltando que estas han sido clasificadas entre las cien empresas más grandes de la industria manufacturera colombiana con mayores ingresos en el 2018. Finalmente, la empresa Mexichem Resinas Colombia S.A.S. es una empresa filial de la empresa química mexicana Mexichem. En este sentido, la filial colombiana no elabora reportes corporativos. 
Las descripciones anteriores permiten concluir que existen tres aspectos principales por los cuales las compañías que han sido catalogadas como grandes empresas de la industria manufacturera en Colombia no han elaborado reportes corporativos. Estos aspectos son: primero, por ser empresas nacionales que pertenecen a grupos empresariales; segundo, por ser empresas controladas por multinacionales; y tercero, por desconocimiento o desinterés de divulgar información no financiera.

Por otro lado, la tabla 4, permite establecer que la segunda categoría con mayor resultado fue la de los Informes de Sostenibilidad, los cuales predominan sobre los Reportes Integrados y, que finalmente, la categoría de Informes de Gestión es la menos usada por las empresas analizadas. De igual forma la tabla 4, permite establecer que empresas como la Sociedad de Fabricación de Automotores S.A.S., Alpina Productos Alimenticios S.A., Colombina S.A. y Colgate Palmolive Compañía se han caracterizado por ser constantes en la elaboración de informes de sostenibilidad durante el periodo analizado.

Por otro lado, empresas como Cementos Argos S.Ay Grupo Argos S.A. se han caracterizado por ser constantes en la elaboración de reportes integrados durante el periodo analizado. Otras empresas como Refinería de Cartagena S.A.S., Cemex Colombia S.A. y Productos Familia S.A. han tenido variaciones en los reportes corporativos que han divulgado, elaborando así, diferentes tipos de reportes corporativos para divulgar su información no financiera.

Ahora bien, en relación con la elaboración y divulgación de reportes corporativos por año, se presenta la tabla 5, la cual fue elaborada suprimiendo la categoría de Reportes no presentados (NP); esto con el fin de identificar los años en los cuales los reportes corporativos tuvieron mayor acogimiento en la industria manufacturera en Colombia. En este sentido, se puede establecer que la elaboración y divulgación de reportes corporativos en el periodo analizado ha sido equilibrada, siendo el 2013 y el 2018 los años con inferiores resultados.

Tabla 5. Caracterización anual de los Reportes Corporativos en las empresas colombianas de la Industria Manufacturera.

\begin{tabular}{cccccccc}
\hline Reporte Corporativo & $\mathbf{2 0 1 3}$ & $\mathbf{2 0 1 4}$ & $\mathbf{2 0 1 5}$ & $\mathbf{2 0 1 6}$ & $\mathbf{2 0 1 7}$ & $\mathbf{2 0 1 8}$ & TOTALES \\
\hline Informe de Gestión (IG) & 3 & 1 & 0 & 0 & 0 & 0 & 4 \\
\hline Informe de Sostenibilidad (IS) & 6 & 9 & 9 & 10 & 7 & 8 & 49 \\
\hline Reporte Integrado (RI) & 2 & 2 & 3 & 2 & 4 & 4 & 17 \\
\hline Totales & 11 & 12 & 12 & 12 & 11 & 12 & 69 \\
\hline
\end{tabular}

Fuente: elaboración propia (2019).

Los datos presentados en la tabla 5, son presentados en la gráfica 1, para mayor comprensión. Allí se puede evidenciar que los Informes de Sostenibilidad son los reportes corporativos que han predominado en la industria manufacturera colombiana. Esto es gracias a los significativos esfuerzos del GRI (Global Reporting Iniciative, 2019) desde comienzos del siglo XXI para que las empresas divulguen información no financiera de cara a los impactos económicos, sociales y medioambientales que estas pueden ocasionar. Por su parte los reportes integrados no han tenido una significativa elaboración y divulgación en la industria manufacturera. 
Elaboración y divulgación de los Reportes Corporativos la Industria Manufacturera colombiana

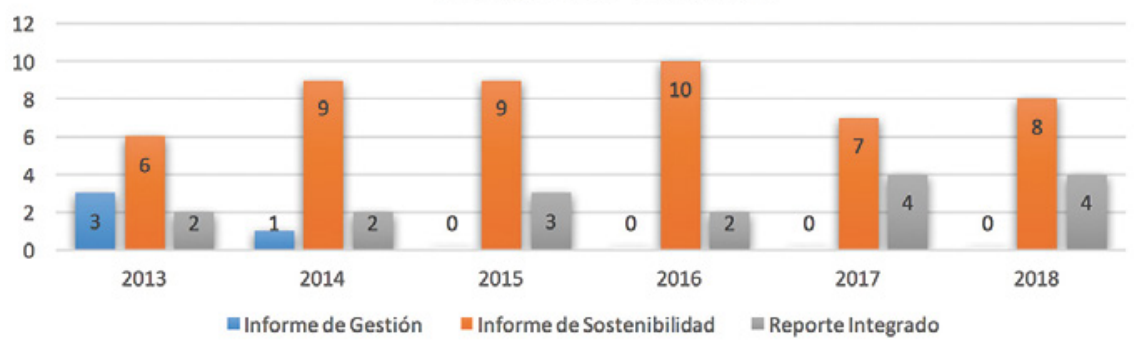

Gráfica 1. Elaboración y divulgación de los Reportes Corporativos en la Industria Minera Colombiana.

Fuente: elaboración propia (2019).

Ahora bien, al revisar la tendencia en la elaboración y divulgación de estos reportes corporativos se presenta la gráfica 2 , la cual permite establecer que los informes de gestión tuvieron un débil acogimiento en el año 2013, y que en los años posteriores su elaboración desapareció. Por su parte, los informes de sostenibilidad para el año 2013, tenían un fuerte acogimiento y en los años venideros su elaboración y divulgación aumentó. Sin embargo, es el año 2017 cuando esta tendencia disminuye y un año después vuelve a aumentar. En relación a los reportes integrados, estos tuvieron mejor acogida en el año 2013 que los informes de gestión. Sin embargo, su crecimiento solo es hasta el año 2017, fecha en la cual los informes de sostenibilidad tuvieron una disminución.

\section{Tendencia de la elaboración y divulgación de Reportes}

Corporativos en el periodo 2013-2016

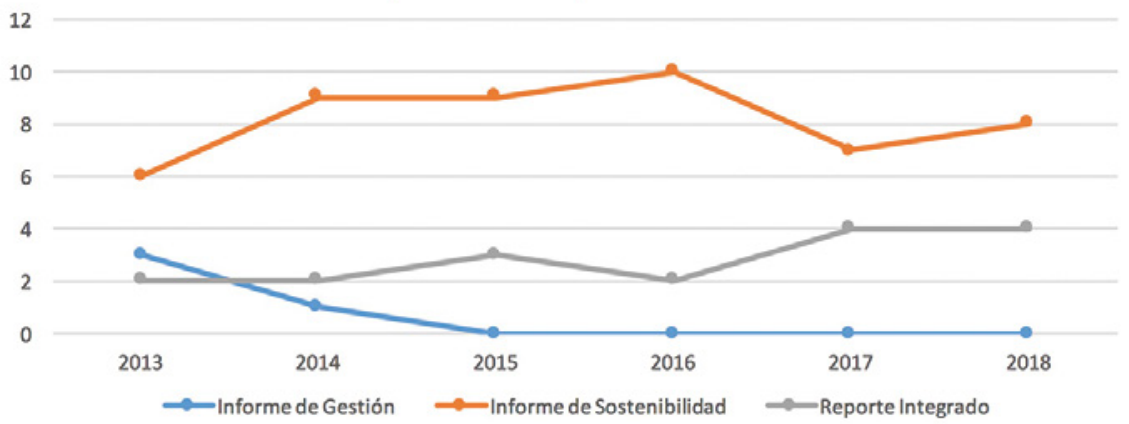

Grafica 2. Tendencia de la elaboración y divulgación de Reportes Corporativos en el periodo 2013-2018.

Fuente: elaboración propia (2019). 
En este sentido, se puede establecer que los informes de gestión han desaparecido del mundo organizacional porque no han logrado responder a las necesidades de información de los diversos grupos de interés. Por su parte, los informes de sostenibilidad han tenido una gran acogida en la industria manufacturera, como se mencionó anteriormente.

Ahora bien, frente al análisis de la elaboración de los reportes integrados se evidencia que son los únicos reportes corporativos que no han presentado disminuciones en el periodo analizado. Esto ocurre por varias razones, entre ellas porque son reportes relativamente nuevos en el mundo organizacional, lo cual ocasiona que las organizaciones deseen acogerlos y adaptarlos como medios de divulgación de información. Adicionalmente, son reportes que reconocen que las organizaciones pueden crear valor en el tiempo para ellas y para los demás a través del reconocimiento del pensamiento integrado en sus operaciones, por medio de su modelo de negocio y del reconocimiento de seis diversos tipos de capital.

\section{Conclusiones}

El presente capítulo permitió establecer que la industria manufacturera colombiana ha presentado un crecimiento económico importante para el PIB del país en relación al año 2018. Sin embargo, existen factores externos que pueden afectar la productividad de esta industria, entre los cuales se destacan: la volatilidad de la tasa de cambio, la calidad de las materias primas, los altos costos de transporte y logística, las altas tasas del sistema tributario colombiano, y no menos importante, la informalidad y el contrabando. En este sentido, la industria manufacturera y el gobierno colombiano tienen grandes retos para mitigar estos factores.

En relación a la elaboración y divulgación de reportes corporativos en la industria manufacturera en Colombia, esta ha sido diversa. La caracterización realizada en este capítulo permitió establecer que la mitad de las empresas analizadas no divulgan información no financiera por tres razones: a saber: i) por ser empresas nacionales que pertenecen a grupos empresariales; ii) por ser empresas controladas por multinacionales; y iii) por desconocimiento o desinterés de divulgar esta clase de información. Esta última razón causa bastantes inquietudes y cuestionamientos al respecto, puesto que las empresas analizadas en este capítulo son consideradas las empresas colombianas más grandes en la industria manufacturera al 2018.

En este sentido, es importante establecer que otra de las posibles razones por las cuales las empresas en Colombia no han generado reportes corporativos es debido a los altos costos que representan para estas su elaboración. De esta forma las empresas que elaboran y divulgan información no financiera por medio de reportes corporativos cuentan con áreas especializadas y equipos de profesionales que se encargan de recopilar la información, para posteriormente representarla en informes con diseños innovadores. Sin embargo, es de aclarar que este argumento no es consecuencia del análisis realizado en la industria manufacturera, puesto que no se tuvo contacto con el personal de las empresas para soportar este argumento.

Por otro lado, los resultados presentados permitieron evidenciar que en la industria manufacturera colombiana la tendencia para elaborar y divulgar información no financiera 
ha estado encaminada hacia los informes de sostenibilidad, pues son estos informes los que lideran este sector económico. Por su parte los reportes integrados han estado presentes en este sector, pero su elaboración aún no ha sido significativa.

Sin embargo, en el año 2017, la elaboración y divulgación de los informes de sostenibilidad disminuyó, y en ese mismo año, la elaboración de los reportes integrados aumentó. Este fenómeno puede estar relacionado con la presunción de que los informes de sostenibilidad serán reemplazados por los reportes integrados, dado que las exigencias del contexto organizacional y de los diversos grupos de interés estarán enfocados a reconocer la creación de valor en las organizaciones por medio del pensamiento integrado y del reconocimiento de los diversos tipos de capital.

Finalmente, se establece que los avances de la divulgación de información no financiera de las empresas colombianas más grandes de la industria manufacturera no han sido significativos, puesto que la mitad de las empresas analizadas no elaboran reportes corporativos por las razones mencionadas anteriormente.

En este punto es necesario establecer que actualmente la elaboración y divulgación de información no financiera para Colombia es de carácter voluntario. Sin embargo, las exigencias de los mercados y las necesidades de información de los diversos grupos de interés provocarán que esta información sea elaborada y divulgada de forma obligatoria, puesto que se hace necesario empezar a llenar los vacíos de información en los cuales la información financiera no ha podido responder, claro ejemplo de esto, son los impactos sociales, económicos y ambientales que causan las empresas, así como también las actividades proyectadas por las empresas para disminuir estos impactos. De esta manera, los desafíos para la industria manufacturera en términos de divulgación de información no financiera son bastante retadores.

\section{Referencias bibliográficas}

Acoplásticos. (2014). Un nuevo impulso a la industria manufacturera colombiana. Panorama, retos y 50 recomendaciones de política.

Acoplásticos. (Octubre de 2019). Nuestra asociación. Obtenido de Acoplásticos: http:// www.acoplasticos.org/index.php/mnu-nos/mnu-nos-nta-aso.

ANIF. (2016). Riesgo Industrial. Asociación Nacional de Instituciones Financieras.

Arraut, L. (2010). La gestión de calidad como innovación organizacional para la productividad en la empresa. Revista EAN, 20 - 41.

Ávila, R. (5 de Mayo de 2019). Las potencias manufectureras. Revista Dinero.

CVN. (Julio de 2019). Industria Manufacturera. Centro Virtual de Negocios.

DANE. (2019). Boletón Técnico - Indice de Producción Industrial (IPI). Colombia: Departamento Nacional de Estadistica.

Delgado, J. y Gutiérrez, J. (1997). Métodos y técnicas cualitativas de investigación en Ciencias Sociales. Revista Andaluza de Relaciones Laborales. 
El Tiempo. (Mayo de 2019). Industria y comercio del pais siguen con racha de buenos resultados. El Tiempo.

EMIS Professional. (Octubre de 2019). Industria Nacional de Gasesosas S.A. (Colombia). Obtenido de EMIS Professional: https://www.emis.com/php/company-profile/ CO/Industria_Nacional_De_Gaseosas_SA_es_1208579.html.

Espinel, P., Aparicio, D. y Mora, A. (2018). Sector textil colombiano y su influencia en la economía del país. Punto de Vista, 1-10.

FAEDIS . (Octubre de 2019). Sector Industrial. Obtenido de Universidad Militar Nueva Granada.

Fedesarrollo. (2019). Prospectiva Económica Abril 2019.

Global Reporting Iniciative. (2019). Global Reporting Iniciative. Obtenido de https://www. globalreporting.org/Pages/default.aspx.

Grupo de Investigación Cultura Emprendedora. (2008). La industria manufacturera colombiana en la economía mundial. Valoración de su potencial de transformación productiva. Bogotá: Universidad de La Sabana.

Portafolio. (Octubre de 2019). Producción industrial creció en febrero. Portafolio.

Procolombia. (Octubre de 2019). La manufactura en Colombia. Obtenido de Procolombia: http://www.procolombia.co/compradores/es/explore-oportunidades/manufacturaen-colombia.

Revista Dinero. (16 de Abril de 2019). Industria avanza a buen ritmo. Revista Dinero.

Revista Dinero. (21 de Mayo de 2019). Industria va al mismo paso del PIB. Revista Dinero.

Superintendencia de Sociedades. (2019). Comportamiento de las 1.000 empresas más grandes del sector real por ingresos operacionales. Bogotá.

Uribe, J. (2014). La industria manufacturera en Colombia entre 2000 y 2013. Revista del Banco de la República, 5-13.

Vicepresidencia de Desarrollo Económico y Competitividad. (2019). Coyuntura económica julio 2019. ANDI. 